Service social

\title{
Éducation populaire et politique : l'urgence d'un renouvellement des modèles traditionnels de la gauche
}

\section{Colette Humbert}

Volume 34, numéro 2-3, 1985

L'organisation communautaire

URI : https://id.erudit.org/iderudit/706275ar

DOI : https://doi.org/10.7202/706275ar

Aller au sommaire du numéro

Éditeur(s)

École de service social de l'Université Laval

ISSN

1708-1734 (numérique)

Découvrir la revue

Citer cet article

Humbert, C. (1985). Éducation populaire et politique : l'urgence d'un

renouvellement des modèles traditionnels de la gauche. Service social, 34(2-3),

308-327. https://doi.org/10.7202/706275ar d'utilisation que vous pouvez consulter en ligne.

https://apropos.erudit.org/fr/usagers/politique-dutilisation/ 
HUMBERT, Colette, directrice de I'INODEP, Paris, France.

\section{Éducation populaire et politique : l'urgence d'un renouvellement des modèles traditionnels de la gauche *}

\section{Colette Humbert}

Le sujet de cette conférence est immense à couvrir et il fait appel à une fresque historico-mondiale, que je dresserai toujours à partir de quelques pays concrets, pays industrialisés et pays du Tiers-Monde, sous l'angle des conjonctures historiques spécifiques à chacun d'eux.

Je souhaite donc seulement partager des constats, quelques éléments d'analyse, une réflexion à partir de pratiques situées en Occident et dans le Tiers-Monde. En aucun cas je ne proposerai de modèles ni de solutions. Tout au plus - et je serai chanceuse, si j'y parviens - j'essaierai d'apporter une contribution à vos débats, à vos interrogations, aux alternatives, plus que jamais nécessaires dans cette sorte d'impasse économique, socio-politique et idéologique dans laquelle nous nous trouvons tous.

Sur les trois aspects que je compte aborder, j'indiquerai d'abord le lieu d'où je parle, ce qui me permettra de situer la problématique et de préciser la définition de l'éducation populaire à partir de laquelle j'aborde le politique. Ce faisant, je toucherai l'articulation entre organisations populaires, d'une part, et partis ou mouvements politiques, d'autre part, incluant les difficultés structurelles et les obstacles au niveau des comportements.

Deuxièmement, je m'interrogerai sur l'avenir du politique dans le contexte actuel : le dire et le faire, le concept de développement, les États-nations et les interrogations aux partis qui ont traditionnellement été des instruments de lutte contre le capitalisme.

En troisième lieu, je parlerai de la difficile question des alternatives : le terrain et la nature du politique, les revendications d'autonomie, les mouvements internationaux. 


\section{Éducation populaire et politique}

\section{L'éducation populaire}

Le lieu d'où je parle, I'INODEP,' 1 est un réseau international d'éducation populaire, d'échanges Sud-Sud, Sud-Nord et de formation dans l'action. Cette éducation populaire se fait dans les organisations que les milieux populaires se donnent; donc autonomes dans leur création, leur direction, leur gestion.

Ces organisations sont de natures extrêmement diverses : défense des droits des paysans en Inde, des femmes en Afrique, des enfants travailleurs en Asie, etc. ; lutte contre des exploitations et dominations précises dans les syndicats de bidonvilles aux Philippines, parmi les groupes de Maoris en Nouvelle-Zélande; amélioration des conditions d'existence par les ligues paysannes d'Amérique latine, les coopératives de planteurs de cacao ou de café au Cameroun, les projets d'autodéveloppement au Zimbabwe; revendication d'identité culturelle sous le thème de la "souveraineté" dans le Pacifique et sous le thème du lien à la terre chez les Kanaks de Nouvelle-Calédonie. Et j'y ajouterai les organisations populaires au Québec, le syndicat autonome des femmes ramasseuses de goémon à l'île-du-Prince-Édouard, et les syndicats du cadre de vie en France.

Ces organisations se sont généralement créées à partir d'une situation-limite ou autour d'un problème (la santé, la terre, l'habitat, le minimum vital, etc.) que des individus isolés ne peuvent pas résoudre. Le mouvement collectif était la seule possibilité de riposte; l'organisation, l'instrument indispensable pour trouver des solutions autres qu'individuelles.

Mais, pour lutter victorieusement, il est indispensable de se former ; c'est la règle de base de l'éducation populaire :

- se former dans l'action et par l'action; analyser des causes qui provoquent, par exemple, le chômage, la baisse de revenu ou l'exclusion sociale. II s'agit là de phénomènes dont les causes structurelles (économiques, politiques et idéologiques) ne sont pas repérables au regard et nécessitent des clés de lecture, des hypothèses de travail permettant de comprendre ce qui se passe et d'agir au bon endroit. Le phénomène localisé (par exemple, le chômage) est le catalyseur de la mobilisation; la visée du combat à long terme, la transformation radicale du système économique ; 
- se former à l'élaboration de stratégies à long terme, ce qui nécessite de connaître les diverses analyses des causes du problème, de repérer des alliés, de créer des fronts et de bâtir des solidarités;

- se former au recueil de données en cours d'action, dans les domaines économiques, sociaux, politiques, historiques et culturels. Elles sont nécessaires, non seulement à la conduite de l'action, à l'analyse indispensable, mais aussi à ce que l'on appelle, en Asie: la construction du "savoir populaire». Ce savoir permet aux milieux populaires de sortir de ce que Paulo Freire nomme la "culture du silence" dans laquelle l'idéologie dominante, l'élitisme organisé, les ont plongés;

- se former pour conduire l'adversaire hors de son propre terrain, d'où il mène le jeu, pour le placer, dépaysé, sur le terrain existentiel des milieux populaires.

Sans revenir sur l'ensemble du thème, je voudrais simplement rappeler trois préalables de la conscientisation conçue comme "pédagogie des opprimés", comme éducation libératrice, comme formation politique. Ces trois points sont une conception de l'homme, de la connaissance et de la culture.

L'homme, être social, est potentiellement sujet de sa vie personnelle et collective. Il doit exister consciemment et en communauté pour se réaliser; il lui faut donc se libérer de ce qui entrave cette " existence-pour-soi ». Il est "être politique» par définition. Comme me disait un Africain : "ma naissance a été mon premier acte politique, parce que je suis né dans un pays, dans une culture, dans une classe sociale et dans un moment historique".

L'homme se caractérise par sa capacité à se distancier de luimême, de son action et de sa réalité, pour l'analyser. Il en découle une conception du savoir, ou plutôt de la connaissance, autre que la conception dominante. Refus du savoir transmis par l'éducation bancaire, des modes formels d'acquisition de ce savoir dispensé par des systèmes, des appareils d'État, des institutions officielles qui disent, imposent ce qu'il faut savoir, pour engager des processus d'acquisition des connaissances dans l'action, à partir des problèmes que vivent les gens et des explications spontanées qu'ils en donnent.

Nouvelle conception de la culture aussi : ni folklorique, ni passéiste, ni réduite à la langue ou accrochée à des traditions à faire revivre à tout prix, mais culture comme "manière spécifique d'être au monde». Freire la définit fort justement comme "l'ensemble cohérent de réponses qu'un groupe humain spécifique donne aux défis qui lui sont lancés par son environnement". 
L'éducation populaire ne peut donc pas être une manière adaptée de faire passer des idées, d'apprendre aux milieux populaires ce qu'ils doivent savoir pour être de bons citoyens, des travailleurs rentables ou des militants dociles; pas plus qu'une "pédagogie" à l'usage de l'ignorance des masses ou, pire, des "masses incultes", comme me disait un jour un intellectuel qui se pensait politisé. Elle est renversement de perspectives et conduit au "savoir utile» pour lutter contre les dominations de toutes sortes.

Une dernière remarque ou un dernier rappel s'impose, pour établir le lien entre éducation populaire et politique. Née il y a une trentaine d'années, en dehors des organisations politiques issues du mouvement ouvrier historique (syndicats et partis politiques de gauche), cette éducation conscientisante s'est surtout développée dans les groupes et organisations populaires autonomes, ayant un souci de participation démocratique maximum des milieux populaires, à la fois dans leur organisation elle-même et dans leur action dans la société.

Il s'agit donc bien d'acteurs sociaux, engagés dans les rapports de force politiques, et luttant pour la transformation - que l'on doit envisager de plus en plus radicale - des structures et des mentalités. C'est bien une pédagogie politique pour une action politique qui lutte contre les exploitations économiques, les dominations politiques, les aliénations culturelles et religieuses.

Je dois cependant ajouter que toute formation dans les organisations populaires n'est pas, de soi, politique, au sens où je viens de le décrire; car deux dangers guettent les noyaux mobilisateurs des groupes conscientisants :

- un "basisme», à la limite de la démagogie (machiavélique ou naive?), qui prétendrait que les milieux populaires "seuls" (sans l'aide de personnes externes ni d'alliés) peuvent à la fois déclencher et mener à bien une transformation radicale des structures et des mentalités. Cela ne s'est jamais vu dans I'histoire (ça se saurait !), ni même dans l'histoire contemporaine. Ce ne fut même pas le cas de la Commune de Paris dont la vie fut si éphémère ;

- le danger de récupération, pas seulement par les pouvoirs en place, mais aussi (et cela se voit) par des organisations politiques de gauche en mal de troupes.

En résumé, l'éducation populaire dont je parle est éminemment politique. Elle se réfère aux grands courants anti-capitalistes dans les pays occidentaux et anti-impérialistes dans les pays du Tiers-Monde. Mais j'insiste : elle a pour point de départ et lieu privilégié d'expression 
les organisations que les milieux populaires se donnent pour améliorer leurs conditions d'existence et pour défendre leurs droits. C'est une éducation/formation dans l'action qui les conduit progressivement à passer des problèmes vécus au dévoilement des conflits d'intérêts de classes, à l'analyse des contradictions structurelles dans la société ; à passer des problèmes locaux aux enjeux nationaux et internationaux.

\section{L'articulation entre organisations populaires et partis ou mouvements politiques de gauche}

L'un des objectifs prioritaires de ce processus d'éducation populaire, politique et conscientisante a toujours été que les organisations populaires s'articulent aux organisations syndicales ou politiques porteuses de changement, afin de rejoindre les fronts nationaux ou internationaux de lutte. Mais cela ne va pas de soi. Les difficultés ne manquent pas. Du fait de leurs natures différentes, il y a des obstacles objectifs à cette articulation; obstacles surmontables, mais dont il faut prendre la mesure. Je ne signalerai que les plus importants.

Concernant le type organisationnel, les partis politiques sont institutionnalisés. Leurs structures organisationnelles et de fonctionnement sont hiérarchisées, la spécialisation des tâches y est grande : en fait, on y reproduit la division du travail sur laquelle reposent nos sociétés occidentales. Et... bien souvent, le carriérisme n'y est pas absent! Les organisations populaires qui ont commencé comme groupes, comités, collectifs, ne présentent pas le même type d'organigramme descendant; elles gardent dans leurs structures mêmes les mécanismes qui permettent des autonomies, des lieux de créativité, et visent au partage maximum des tâches et des responsabilités.

Concernant le champ d'action, les organisations politiques le situent d'emblée au niveau macro (national et souvent international), alors que les organisations populaires sont engagées au niveau micro (local ou sectoriel), dans la quotidienneté de la vie. Les liens avec le national et l'international sont un des axes de leur formation, mais le passage ne se fait pas ipso facto, d'autant plus que les partis sont souvent peu engagés dans leurs luttes sociales.

Concernant le type de pouvoir interne, les partis (même ceux qui se réclament de l'autogestion en France) ont une direction centralisée autour d'un projet national, et ont un côté "entreprise", avec des échelles de salaires qui ne sont pas toujours sans poser problème. Dans les organisations populaires, les prises de décisions sont partagées avec et par les militants ou groupes et leaders de la population. Il y a plus 
souvent consultation de la base sociale, donc tentative de démocratie directe.

Concernant l'appartenance sociale des membres (composition sociale interne), il faut voir que les partis à référence socialiste sont souvent inter-classistes ou constitués en majorité de représentants des classes moyennes, notamment d'intellectuels solidaires des classes populaires, au niveau de l'idéologie et des programmes qu'ils avancent. Et, à cet égard, les proportions varient selon les partis. II faut faire exception cependant pour les partis communistes européens, construits par des ouvriers, composés d'ouvriers, et qui, en France et en Italie, ont su rallier jusqu'à six millions d'électeurs, soit autour du quart de l'électorat de leurs pays respectifs. Dans les organisations populaires on retrouve, par définition, des représentants des classes populaires issus de catégories et couches sociales spécifiques, surtout des chômeurs, des assistés sociaux, et des marginalisés, regroupés autour d'objectifs précis.

Concernant le type de formation, les partis la font à l'intérieur même de leurs structures, leurs écoles, leurs sessions, leurs congrès. Bien sûr, dans des actions de masse également (quand elles existent) ; mais le processus va généralement de la théorie à la pratique, en passant par les programmes. Pour ce qui est des organisations populaires, nous avons vu que la formation s'y réalise dans l'action et donc que le processus est inverse: de la pratique aux théories. II y a élaboration des concepts, recours aux analyses des partis ou aux grands courants idéologiques.

Il s'agit là d'éléments objectifs qui, en eux-mêmes, comportent des difficultés pour l'articulation organisations populaires/partis. Mais cela fait précisément partie de la formation politique que d'apprendre à les surmonter. Les partis ont un gros effort à faire en la matière. Sans compter que les organisations populaires elles-mêmes ne sont pas à l'abri de la reproduction des modèles institutionnels de nos sociétés. II faut beaucoup de vigilance pour que les leaders populaires ne reproduisent pas les pratiques de pouvoir que nous connaissons bien. II faut beaucoup de vigilance à l'égard des alliés petits-bourgeois intellectuels pris entre deux tentations: celle de parler pour le peuple, d'imposer leur langage et leurs analyses ou celle de porter leur coeur en berne (ils "se sentent mal») parce qu'ils ne sont pas sortis de la confusion entre leur option de classe et leur situation de classe. Une organisation populaire n'a tout de même pas pour vocation d'être le lieu de leur thérapie!

Une fois les obstacles structurels identifiés, il faut parler d'autres obstacles, à la fois plus faciles à gérer, parce qu'ils ne relèvent pas de la 
nature même des organisations dont nous parlons, mais aussi plus difficiles à reconnaître (et à admettre) parce qu'il s'agit de pratiques internes aux partis: le niveau de langage, les comportements, en un mot l'habitus des professionnels politiques.

On ne peut le nier, comme dans toute institution, il y a un langage de parti, et de parti de gauche. Ce langage d'intellectuel est souvent un code fermé par lequel on se protège, et qui ne fonctionne qu'entre initiés. Quand il s'agit d'un parti qui se dit "solidaire des milieux populaires", qui a opté pour un projet de société dans laquelle ces milieux populaires seront acteurs à part entière, il se disqualifie : s'il produit des textes publics parfaitement incompréhensibles pour une assistée sociale ou un chômeur; et si, dans les débats auxquels il invite les milieux populaires, la dynamique des grandes tirades oratoires et les citations des grands classiques laissent indifférents ceux qui sont concernés au premier chef.

Or, le langage, ce n'est pas seulement des mots, des agencements de mots, une manière d'exprimer des idées. C'est aussi l'expression d'une culture et, dans ce cas précis, l'expression d'une culture dirigeante, plongeant ceux qui n'en sont pas, une fois de plus, dans la culture du silence. J'ai entendu des envolées lyriques partant de la plus-value pour aboutir au front de masse. Les masses n'y ont rien compris, d'autant plus, d'ailleurs, qu'elles auraient refusé d'être qualifiées de "masses".

Les intellectuels de gauche sont des verbo-moteurs. Ils parlent, ils parlent! Rien n'égale leur habitude de la parole et la jouissance qu'ils en tirent! Ce qui les empêche de voir qu'il n'y a plus ni moment, ni espace, ni possibilité pour une assistée sociale ou un ouvrier de s'exprimer; encore moins de s'approcher d'un micro. II y a bien deux cultures en présence: celle d'un langage articulé, enraciné dans l'intellect, et celle d'une parole existentielle qui s'articule en se disant et qui origine du cœur et des tripes. Et ne parlons pas de la monopolisation des débats par la maîtrise des règles de procédure...

Je me retrouvais récemment dans un groupe de travail confronté justement à ce thème: "milieux populaires et experts : deux cultures en opposition?" alors que, dans un groupe de ressources techniques (G.R.T.) on s'interrogeait sur: "comment, à partir des problèmes d'habitat, rejoindre un projet de société".

Si l'on évoque le Tiers-Monde, on prend la mesure de la révolution culturelle et politique qui est à faire dans les partis qui se réclament du marxisme. Il y a beau temps qu'on y a dénoncé le langage de bois des avant-gardes éclairées (c'est ainsi qu'elles se voient). On y a dénoncé la culture internationale de gauche qui ne tient pas compte des situations 
concrètes des paysans, des chômeurs et des travailleurs en situation précaire. On y a dénoncé une certaine culture politique où les concepts tiennent lieu d'analyse et où les slogans, préfabriqués ailleurs, sont les moyens par lesquels on prétend mobiliser le peuple; mais par lesquels, en réalité, ces prétendus maîtres de la révolution restent les maîtres du peuple.

Il faut avoir vécu une autre approche de la communication avec le peuple pour comprendre la différence. Je me souviendrai toujours de cette longue soirée de discussion avec des femmes de l'île-du-PrinceÉdouard, au cours de laquelle était évoquée l'augmentation du prix des diverses pièces d'équipement (tabliers, râteaux, bottes, etc.) dont elles ont besoin pour l'exercice de leur métier de ramasseuses de goémon (d'où est tirée une substance utilisée dans des produits alimentaires, pharmaceutiques, etc.). Après qu'elles eurent pris conscience, ensemble, que leur travail était une production, l'une d'elles se mit à parler de "coûts de production" en rapport avec l'augmentation du prix de l'équipement. Le concept intervenait au bon moment du processus de connaissance, pour nommer une dimension centrale de la pratique économique de ces femmes, dimension réellement perçue et reconnue par toutes. Qui soutiendra que le concept aurait eu la même portée s'il avait été introduit au point de départ, comme cadre de la discussion ? Les femmes de l'île l'utilisent maintenant couramment dans leurs négociations sur le prix du goémon avec les multinationales.

Que les classes dominantes du capitalisme (national et international) continuent à prétendre qu'elles feront le bonheur des gens s'ils sont bien dociles, bons élèves et sages, on ne s'en étonnera pas. Mais que des partis de gauche chantent la même chanson, sur un autre air : "suivez-nous, nous savons ce qu'il faut faire, faites-nous confiance, demain tout sera changé", cela est intolérable! Il s'agit là d'attitudes "paternalistes" ou élitistes qui considèrent le peuple comme incapable de penser et d'agir. Il s'agit de comportements technocratiques ou bureaucratiques qui révèlent à quel point l'idéologie dominante a pénétré dans les mœurs de ceux-là mêmes qui la combattent.

Partis et organisations populaires doivent donc mutuellement se questionner. Les partis interrogent les organisations populaires sur leur capacité à entrer, au niveau national, dans les rapports de force et les conditions réelles de lutte ; sur leur capacité à prendre en compte, tout au moins à ne pas ignorer les analyses proposées par les organisations politiques. À leur tour, les organisations populaires interrogent les partis sur leur capacité de formation, leur pédagogie politique, leur enracinement dans les classes et la culture populaire. C'est ce dont parlait Mao lorsqu'il souhaitait que "le peuple soit la conscience 
critique du parti, et le parti, la conscience critique du peuple». Double interrogation indispensable à la naissance d'alternatives.

\section{Quel avenir du politique?}

II y a vingt ans, nous étions en pleine euphorie. Presque tous les pays colonisés avaient accédé ou accédaient à l'indépendance, l'antiimpérialisme mobilisait les pays du Tiers-Monde. Les partis de gauche, en Europe surtout, avaient le vent en poupe, on parlait de l'eurocommunisme à visage humain, de la social-démocratie, et certains partis socialistes semblaient promis à un bel avenir. Ici, au Québec, les groupes populaires se développaient, le syndicalisme jouait un rôle important d'analyseur politique et faisait des conquêtes en faveur de la classe ouvrière; par ailleurs, la grande espérance d'un peuple se levait.

Or, en 1985, que constate-t-on? l'arrêt brutal de l'abondance économique promise, le demi-échec des gouvernements socialistes, la consolidation des dictatures militaires dans les pays africains, les situations de faillites économique et financière des nouvelles démocraties d'Amérique latine et leurs difficultés énormes à reconstituer le tissu social détruit par les années d'exercice du pouvoir par des dictatures militaires; l'anti-communisme primaire relève la tête, la Chine populaire s'ouvre à l'Occident hier haï, le désenchantement politique gagne beaucoup de militants.

Alors? Avenir bouché? On baisse les bras? Ou, avec ceux qui commencent à le faire, est-ce le moment de remettre en cause à la fois la nature, le champ et les pratiques du politique, pour mieux cerner ce que peuvent être des partis ou mouvements porteurs de changement, les pratiques à mettre en place, les nouvelles formes d'éducation à innover? Sommes-nous devant un vide ou devant un espace ouvert à la créativité politique?

\section{Le dire et le faire politiques}

Une grande question domine notre époque, à la fois unitaire et émiettée, centraliste et centrifuge ; les uns rêvant d'un gouvernement mondial, les autres d'un localisme préservé. En effet, il semble que tout s'est fait comme si le discours politique pouvait se dispenser du rapport au réel et au quotidien; actuellement, cela est aggravé par les sondages d'opinion et le marketing politique qui semblent de plus en plus dispenser de "faire». Or, "être révolutionnaire, disait Gramsci, c'est agir sur la réalité». 
Le dire actuel des partis traditionnels de gauche se cramponne aux rigidités doctrinales et semble cultiver la paresse de l'esprit (on répète sur le mode incantatoire), ou "on n'a plus rien à dire puisque c'est la crise!». Créé en 1920 par une poignée de bolchéviques, le Parti communiste français ralliait, au début des années soixante-dix, le vote de six à sept millions d'électeurs, essentiellement des ouvriers. Il n'aura fallu que quinze ans pour passer de ce succès à la désagrégation actuelle. Encore au dernier congrès, ceux qu'on a appelés les «novateurs" ont échoué dans leur tentative pour enligner le parti sur le moment historique actuel. D'où la désaffection des milieux populaires qui en ont assez d'être menés en bateau et en appellent au faire : un faire qui doit répondre rapidement et avec justesse à leur impatience et à leur grande espérance trahie. "Changer de vie? changer la société ? oui, mais aujourd'hui et pas demain!"

Le faire est la première condition de la crédibilité. Le problème qui se pose n'est pas celui de renforcer ou d'affiner les théories, mais celui d'une action politique enracinée dans les réalités quotidiennes des milieux populaires.

Qu'on ne se méprenne pas sur la signification de ce faire. Tout ne changera pas tout de suite. II est bien clair qu'un projet politique est nécessaire et qu'une action de transformation radicale des structures et des mentalités est soumise à trois grandes contraintes. Toute politique nécessite un coût : le tout est de savoir comment, pour qui, sur quoi (on l'a bien vu, en France, avec la question de l'impôt sur les grandes fortunes). Elle demande aussi du temps pour se mettre en place et produire des effets. Toute stratégie de changement social se heurte actuellement à la chaîne macro-économico-technique qui étouffe le monde, y compris les pays qui se disent socialistes.

Le faire exige enfin une démarche politique nouvelle face à deux obstacles que constituent la conception du développement des pays occidentaux, basée sur la croissance économique, et la structuration du monde en États-nations. Ces deux points centraux à prendre en compte vont bouleverser notre conception du politique et celle des partis eux-mêmes : ils conduisent à réinventer le socialisme ou, plus exactement, "les socialismes".

\section{Repenser le développement}

Il y a fort longtemps que les Occidentaux confondent l'évolution de leur société avec celle de l'humanité entière. Ils en ont ainsi faussé les conditions et les possibilités d'évolution. Le développement doit cesser d'être le processus technico-économique par lequel les sociétés 
conquérantes ont asservi le monde et l'ont façonné à leur image. La dynamique concurrentielle sur laquelle repose notre système est par essence non maitrisable et a abouti à une perversion: désormais, l'économie, avec sa dimension technique, commande tout le reste: le politique, le social, le culturel. Les pays industrialisés absorbent la quasi-totalité des ressources valorisables dans le monde; ils détiennent le monopole du travail. Or, à l'échelle du monde, les ressources ne sont pas inépuisables et on connait les cris d'alarme qui montent d'un peu partout. Par ailleurs, le fossé se creuse dangereusement entre les besoins des pays consommateurs et les ressources disponibles à moyen terme. Le Rwandais ne pourra jamais vivre, un jour, comme l'Américain moyen qui consomme actuellement mille cent fois plus d'énergie que lui. L'internationalisation et la concentration du capital ont fait que toutes les nations ont contribué à l'envoi de l'homme dans la lune et à la fabrication de missiles nucléaires, tandis que la faim revient en force dans les pays du Tiers-Monde. C'est alors qu'on nous promet un nouveau "siècle des Lumières"; avec le paradis des "tiques". On attend tout du miracle de la robotique et de l'informatique, dont le premier effet est l'élimination du "travail vivant", ici, mais surtout dans les pays du Tiers-Monde; travail vivant qui, avec les ressources naturelles était leur grande richesse. En l'an 2000, le Gabon verra ses ressources en bois et en fer épuisées, et il sera l'un des peuples les plus pauvres du monde, sans parler de sa dette.

Les pays industrialisés, pour maintenir leur niveau de vie, sont condamnés à la fuite en avant: production, technologie, finances; cycle infernal d'une construction technico-économico-mondiale qui court à sa perte. D'autant plus que les méga-outils de la puissance créent des rigidités de plus en plus coûteuses à contourner. Les systèmes techniques sont devenus tels, que les adapter à un élément perturbateur est devenu plus long que le délai moyen séparant une première perturbation de la deuxième. La tragédie de Bhopal, en Inde, n'était pas encore sortie de l'actualité que déjà Union Carbide se voyait confrontée à des difficultés semblables aux États-Unis. "Les outils industriels actuels attirent les catastrophes !»

L'organisation socio-politique qui en découle repose sur le meurtre de classes sociales et de peuples entiers. Cette atroce injustice qui a rendu possible, jusqu'ici, le "système technicien", nous conditionne complètement. Nous ne pouvons pas envisager - théoriquement - de réelles alternatives. Les possibilités sur ce plan seront - ici et maintenant - pragmatiques. Et c'est bien ce qu'elles sont, nous le verrons plus loin. 
Le développement matériel, tel qu'il existe en pays occidental, est impossible à l'échelle de l'humanité ; il est socialement inacceptable et, de plus, c'est une faillite financière. Plus grave: le champ du politique et celui de l'économique ne coïncident plus du tout: le politique demeure national, l'économique devient de plus en plus international.

Mais le capitalisme n'a pas seulement perverti le politique. Son histoire est celle de la dépossession qu'il opère des capacités autonomes des hommes, des femmes et des sociétés, pour en faire des sources de profit: santé, loisirs, habitat, etc. C'est bien dans sa logique propre, encore, de tout transformer en "marchandise». L'expansion du capitalisme a tué la créativité dans la majeure partie de l'humanité, y compris dans nos sociétés techniciennes qui la réservent à une minorité de plus en plus étroite et concentrée; d'où une disqualification de plus en plus grande des travailleurs et du travail : la fonction remplace de plus en plus le métier, et les techniques le savoir-faire.

Que faire alors de ceux qui n'ont plus et n'auront plus accès au travail? Ce n'est pas par hasard si, dans les sociétés libérales ou néolibérales avancées, on en appelle aux "solidarités premières", à la nécessité de se prendre en charge ; si l'on évoque :

- l'économie duale : avec coexistence au sein de chaque pays de l'économie hautement sophistiquée, performante, bien rémunérée, et de l'économie informelle permettant la survie des rejetés du système et la reproduction sociale ;

- la société dualiste dans laquelle chaque travailleur et travailleuse partagerait son temps entre la production intégrée et la production autonome. C'est la sortie obligée, la parade nécessaire pour éviter l'explosion sociale!

Mais que voit-on dans le Tiers-Monde et qui peut nous menacer à moyen terme? L'économie n'y est pas duale: elle est multiple. Il y a une économie capitaliste. En Inde, par exemple, elle ne touche que $10 \%$ d'une population de 750 millions d'habitants. Et on pourrait faire des constatations analogues pour l'Afrique et l'Amérique latine. II y a une économie de subsistance, qui n'est que régression de l'ancienne économie autonome. Il y a aussi une économie souterraine à multiples faces : chômeurs qui produisent et échangent entre eux, petits métiers (récupération des déchets industriels, transports pour le commerce). C'est une économie de sous-traitance à bon marché. $I 1$ y a enfin une économie clandestine, qui est le terrain de la prostitution, de la contrebande, des trafics illégaux, etc. On comprend dès lors que, de 
plus en plus, on dise: "le développement, c'est tout ce qu'on fait contre le développement".

\section{Les États-nations}

En Occident, l'État-nation remonte à des origines lointaines, et il est longtemps resté circonscrit. C'est un phénomène de centralisation de tous les pouvoirs, sacralisés soit dans la personne du roi, comme dans l'ancienne France ou soit, comme actuellement, dans l'État. Or, toutes les décolonisations ont abouti à l'instauration d'États-nations qui ont laminé les peuples. L'État est devenu l'instrument total dont les révolutionnaires ont rêvé le dépérissement, mais qui ne fait que se renforcer en se complexifiant, se bureaucratisant et, souvent, se militarisant.

Ces deux problèmes, développement et États-nations, interrogent les partis traditionnels de gauche et ceux qui tentent de naître.

\section{Interrogations aux partis traditionnels de gauche}

Nous devons faire un constat. En effet, le socialisme comme le capitalisme sont tous deux nés en Europe. Ils sont partie intégrante de l'idéologie occidentale qui pense incarner l'avenir de l'humanité et voit l'évolution humaine comme unilinéaire. Son critère d'appréciation des degrés d'évolution est, à la fois, la connaissance (assimilée à la seule connaissance scientifique) et la maîtrise des techniques : deux critères qui fondent "faussement" la supériorité de l'Occident ! Le socialisme, qui s'est forgé en opposition au capitalisme, a trop souvent entretenu l'idée que le progrès social ne peut résulter que d'une croissance de la richesse d'une société. Les pays socialistes se sont efforcés d'être compétitifs avec les pays capitalistes industrialisés en ayant recours aux mêmes technologies, en adoptant par conséquent les mêmes normes de production donc en acquérant une capacité de destruction égale à la leur. On peut se demander si cela ne confirme pas et ne conforte pas l'organisation technico-économique du monde, et s'il sera encore longtemps possible d'expliquer en termes idéologiques l'actuelle division politico-militaire du monde.

Quant aux partis traditionnels de gauche des pays occidentaux, ils se sont résolument placés sur le terrain économique, mettant à leur programme les nationalisations de l'appareil de production (sans remettre en cause la production) et une plus juste distribution des fruits du travail (sans assez remettre en cause l'origine de ces profits : le pillage du Tiers-Monde par exemple). 
Dans les pays du Tiers-Monde, les partis à référence socialiste se sont placés dans l'obligation de jouer à fond la carte économique pour se donner la marge de manœuvre qui leur permette de jouer la carte politique. En 1969, j'ai fait partie d'une mission auprès du Ministère du plan de l'Algérie de Boumedienne. L'option du ministre en titre était claire: miser à fond sur le développement de l'industrie pétrolière pour créer une richesse qui rende possible le développement dans les autres secteurs, et notamment le développement social. L'horizon du plein emploi était alors fixé à 1986. Resterait à aller voir si le plein emploi y est effectivement atteint à ce jour.

Dans les pays industrialisés, les partis traditionnels de gauche ont revendiqué pour tous les avantages que les classes dominantes avaient annexés pour elles, à partir de l'accumulation des richesses. Ce furent de réels acquis, au terme de luttes rudes et longues. Mais on ne peut en rester là !

Nous référant à ce qui vient d'être évoqué, il manque, de toute évidence, une réflexion sur ce qu'est le développement des sociétés dans toutes leurs dimensions: le culturel (les valeurs que l'on privilégie), le politique (en termes de sens: direction et signification de rapports sociaux), tous deux déterminant l'économique.

De plus, les partis de gauche, et aussi bien les syndicats, ont développé un discours et une pratique de partenaires de l'État. Ils ont visé à occuper l'État, sans en remettre vraiment en cause la nature, dont ils héritaient ou qu'ils voulaient investir. Et l'on a vu s'instaurer des capitalismes d'État, des socialismes à la suédoise où l'être humain est pris en charge de la naissance à la mort, ou des socialismes, comme en France, gestionnaires de la crise. Enfin, ces partis, au lieu d'être des instruments de transformation des structures et des mentalités, sont devenus ou deviennent des courroies de transmission entre l'État et l'électorat. Finalement, ils ne sont que des agents d'amélioration des conditions de vie des classes moyennes ou de la petite-bourgeoisie. Et, objectivement, ils deviennent des alliés du capitalisme. Ce qui est contre leur nature même. C'est leur grand drame, et celui de la socialdémocratie en particulier qui, au départ, dans l'Europe d'avant 1914, s'appuyait sur deux principes de base: triple réseau organisationnel partis/syndicats/coopératives et associations ouvrières, dialectique entre le mouvement ouvrier et les théories marxistes. On voit bien l'abandon qui s'est fait, depuis, des associations ouvrières, du projet politique des coopératives, et comment la théorie a pris le pas sur les pratiques populaires et ouvrières.

Aujourd'hui, cette articulation doit se retrouver pour que les partis puissent se concevoir comme des instruments d'animation et de 
transformation de la société, tout en sachant bien que tout ne passera pas par eux. Ils ne devront pas régenter une société ou l'expérimentation sociale de nouveaux noyaux d'acquisition de connaissances jouera un rôle moteur. Affirmer qu'une nouvelle culture politique est nécessaire dans l'impasse actuelle c'est se mettre, ipso facto, dans la nécessité d'intervenir ici et maintenant. C'est, comme on l'a vu écrit sur les murs de la Sorbonne en 1968, "faire place à l'imagination». Plus que jamais l'imagination est nécessaire alors que, dans le même temps, elle est tuée par notre société technicienne qui nous dit que "la mer" - lieu de rêve, de beauté, de dépassement - n'est que de "l'eau salée", pire "un mélange $\mathrm{d}^{\prime} \mathrm{H}_{2} \mathrm{O}$ et de $\mathrm{NaCl}$ ". II faut l'affirmer: en définitive, ce seront les peuples qui feront l'histoire et non pas les financiers de Wall Street ou l'empire McDonald! Et l'on voit apparaître, ici et là, dans le Tiers-Monde et en pays occidentaux, les signes avantcoureurs d'une histoire nouvelle qui commence à s'écrire.

\section{Les alternatives}

Je retiendrai trois principaux éléments nouveaux porteurs d'alternatives:

- le terrain et la nature du politique sont en train de changer ;

- les revendications et les pratiques d'autonomie;

- l'articulation des mouvements sociaux et des organisations politiques.

\section{Le terrain et la nature du politique sont en train de changer}

En ce qui a trait au terrain politique, sans lâcher l'analyse du capitalisme et de l'impérialisme, il y a retour concret au symbolique comme expression du vécu. L'appel à la culture, à d'autres valeurs et à d'autres comportements sont des mobilisateurs politiques. J'en donnerai deux exemples: la lutte des Kanaks de Nouvelle-Calédonie et un séminaire international de l'INODEP.

Les Kanaks de Nouvelle-Calédonie traduisent bien l'énorme revendication culturelle du Tiers-Monde, sa nouvelle manière d'aborder le politique. Les Kanaks sont les autochtones de Nouvelle-Calédonie. Ils comptent pour $36 \%$ de la population, alors que les colons français, les Caldoches, comptent pour $25 \%$, le reste de la population étant principalement composée de Polynésiens, importés pour le travail dans les mines de nickel. Bien sûr, le Front de libération nationale des 
Kanaks (F.L.N.K.S.) lutte directement contre le néo-colonialisme français. Bien sûr, fait-il l'analyse du capitalisme dont est victime le peuple Kanak, mais l'enracinement de sa lutte est bien culturel, centré autour du problème de la terre. Les mines de nickel ont éventré la terre des Kanaks et cette image a pour eux un puissant pouvoir évocateur et mobilisateur. Tjibao, leader du F.L.N.K.S., disait "La terre est une marchandise, objet de spéculation, mais surtout la terre est "éventrée", "violée", alors que nous sommes les fils de la Terre». En effet, les Kanaks ne se sentent pas fils de la lignée (comme dans la culture arabe, par exemple, où le terme "ben " dans les noms de personne, renvoie à la lignée mâle), mais fils de la Terre. "C'est notre identité culturelle qu'on a tuée, violée", ajoutait Tjibao. Le langage qui nomme la lutte n'est pas ici le "langage de bois" de la culture universelle de gauche, mais le langage existentiel de la culture vécue et sentie dans les tripes du peuple. C'est ainsi que les Kanaks vivent la revendication de la "souveraineté» des peuples autochtones du Pacifique, qui se développe de Hawaï à l'Australie.

Mon deuxième exemple est tiré de la préparation du séminaire international de l'INODEP, à Hyderabad, Inde, en novembre 1985. La conception et les objectifs principaux en ont été écrits par un équipier créole de l'INODEP, avec des femmes maoris de Nouvelle-Zélande. Le thème central est l'éducation politique des milieux populaires à partir de leurs univers culturels et religieux spécifiques. La conception du séminaire part de la constatation que :

Les concepts de développement et de libération tels qu'ils sont mis en pratique dans les pays du Tiers-Monde ont été jusqu'à présent fondamentalement imprégnés de préoccupations occidentales. Toutes les institutions culturelles traditionnelles d'une société sont traitées avec méfiance, mépris ou complète indifférence. Très souvent, le processus de développement cherche à détruire toute la culture d'une communauté. Même parmi les partis politiques progressistes de beaucoup de pays du Tiers-Monde, la différence est infime. Ils semblent avoir accepté le modèle " universel» de développement.

Les objectifs principaux sont les suivants :

Exploration approfondie de l'expérience des participants qui travaillent sur des concepts de culture indigène et sur l'analyse internationale. Autrement dit, une approche sur le travail social et politique engagé dans la libération personnelle et collective, et luttant contre les exploitations de toutes sortes (économiques, politiques, culturelles, technologiques, etc.).

Exploration de la signification des idées locales de justice et de libération que l'on trouve dans les différentes cultures des participants. 
Partage des luttes populaires dans le monde industrialisé, en particulier dans leur combat contre la menace nucléaire et l'étude de ses implications dans le Tiers-Monde.

Ebauche d'une interaction et d'un processus d'apprentissage hors des chemins de la culture dominante.

Par ailleurs, un fait nouveau est apparu, non négligeable, les libérations d'aujourd'hui (même quand elles se sont perverties, parce que récupérées par des pouvoirs dominateurs religieux) ont pris comme point de départ les "résistances culturelles": Iran, Pologne, etc.

En ce qui concerne la nature du politique, de nouvelles pratiques se font jour en Europe: nouvelles formes d'organisation du travail, pratiques autogestionnaires concrètes qui, il y a quelques années, étaient formulées en termes politiques et en références dogmatiques. On est passé du dire au faire. De nouvelles manières de vivre ensemble apparaissent dans des cités ouvrières d'Italie. Des exigences de cohérence s'affirment entre le projet de société dont on est porteur et la vie personnelle. C'est une préoccupation permanente en Belgique dans ces universités populaires que sont les lieux de travail et de vie.

Ces nouvelles pratiques sont d'abord expérimentation avant d'être discours. AUTREMENT! est leur maître-mot. Et il ne s'agit pas là de groupes a-politiques qui se mettent en marge, comme ce fut le cas dans les années soixante et soixante-dix. Ce sont de petits paysans qui, confrontés à un problème, entendent le résoudre en décidant ici et maintenant. Ce sont d'anciens militants d'extrême-gauche qui n'ont pas jeté leurs analyses et leurs grands idéaux aux poubelles de l'histoire, mais qui entendent apporter leur contribution à l'expérimentation sociale. Tel ce groupe, au Mans, composé de militants intellectuels d'origine paysanne qui, décidant de militer autrement, sont redevenus paysans, ont abandonné leurs livres du savoir politique et, entre eux, à partir de ce qu'ils vivent et font au niveau local, se forment à l'analyse de l'impact de leurs luttes contre l'agro-alimentaire, évaluent l'écart entre l'image qu'ils ont d'eux-mêmes et l'image que les populations en ont, entrent dans le réseau européen des travailleurs paysans anti-productivistes.

La lutte pour une véritable émancipation vis-à-vis du système économique passe de plus en plus par le maintien, la resaisie, le renforcement des capacités autonomes, à la fois moteur de toute résistance et moyen situé au-delà de la résistance. II y a réappropriation et amélioration du savoir-faire ouvrier et paysan. Dans les A.C.P. 
(groupe de pays d'Afrique, des Caraïbes et du Pacifique), soixante-dixsept pays ont fait face à la C.E.E. (Communauté économique européenne) pendant un an, à Bruxelles, dans le cadre des négociations pour les accords de Lomé III, avec l'appui de Pisani et la présence solidaire des organisations de paysans travailleurs et des organisations tiers-mondistes d'Europe. L'accent est mis sur l'autosuffisance alimentaire, l'accord harmonieux de la "main et de l'outil». Au Burkina Faso, par exemple, tout un processus de conscientisation s'élabore autour d'une expérience d'élevage de volailles dans des coopératives de production, qui permettent aux paysans de sortir de la grande économie marchande, de vendre directement aux consommateurs ou de réaliser des échanges avec eux. Au plan économique, ils vivent un apprentissage de la gestion autour de la question de la distribution et de l'invastissement des profits. Au plan socio-politique, ils expérimentent l'organisation du travail, le partage des tâches et des responsabilités, l'identification des problèmes. Au plan culturel et idéologique, l'impact se remarque dans tout le village, à travers le sens donné à l'autonomie retrouvée.

\section{Les revendications d'autonomie}

II semble qu'il commence à y avoir fractures dans la construction socio-économique mondiale; on en voit la trace chez certains peuples minoritaires au sein des pays industrialisés (Basques, etc.) et dans les revendications amérindiennes dans les deux Amériques. Au début, ces peuples avaient le souci de revendiquer leur langue et leur patrimoine culturel. Actuellement, ils vont plus loin, refusant les disqualifications, les désertifications, les marginalisations. En France, on peut identifier trois cent cinquante pays ou communautés sociologiques, dont trentesept en Bretagne seulement. Le slogan, en France, c'est d'abord : vivre et travailler au pays maintenant, puis... décider au pays. La question du pouvoir est posée. On retrouve là ce qui concerne le Tiers-Monde : la pluralité des avenirs possibles et la diversité des choix de développement qui permettrait une diversité des modèles.

Partout dans le monde, les jeunes revendiquent leur classe d'âge comme acteur social autonome à part entière. Et, dans chaque pays, ils reconnaissent leur ressemblance avec les jeunes du monde entier : "le béton, les élections, les feuilles de copie, les candidats maussades, les oiseaux malades de pollution : ras le bol! À nous de refaire le monde". 


\section{Les mouvements sociaux}

Pour le moment, ils restent trop souvent des nébuleuses constituées d'éléments hétérogènes parfois contradictoires, manquant souvent d'analyse globale de la société. On a eu raison dans les partis et dans les organisations populaires de s'inquiéter de certaines de leurs naïvetés ou de leur particularisme qui ne prenait pas en compte le tout. Chacun appréhende et analyse la réalité en adoptant un angle d'observation qui fait apparaître comme primordial un aspect particulier de la totalité : rapport de l'homme à son environnement (écologistes et antinucléaires), entre les sexes (féministes), entre pays industrialisés et Tiers-Monde (tiers-mondistes). Puis, à partir de cet aspect qu'il privilégie, chacun lutte, à sa manière, pour transformer ces rapports. Ces mouvements contestataires parviennent ainsi à réunir beaucoup de gens qui ne poursuivent pas les mêmes buts. Mais des secteurs de ces mouvements eux aussi revendiquent le politique et n'en restent pas au particulier. Ils traduisent ainsi un considérable changement d'attitude à l'égard du champ et des organisations politiques. Ils élargissent le débat social et participent à l'élaboration d'un nouveau type de société. Les partis ne s'y sont pas trompés, tel le Parti communiste italien (P.C.I.) qui, il y a quelques années, bannissait Macciocchi pour s'être engagée avec les jeunes de Bologne et qui, aujourd'hui, prend en compte les mouvements sociaux et, dans un prochain congrès, pense à changer de nom, pour s'appeler (peut-être), "Parti populaire démocratique».

Je voudrais faire mention ici de ce qui se met en place en Asie: l'Internationale des enfants travailleurs, qui dès l'âge de six ans, travaillent dans les multinationales, du décorticage de la crevette au montage des micro-processeurs, en passant par la confection de bougies. Ils s'organisent pour survivre, se défendent, et les partis politiques, syndicats et mouvements sociaux se sont faits leurs alliés. Ils travaillent à la reconnaissance des enfants au travail par le Bureau international du travail (B.I.T.), à la défense de leurs droits par des réseaux d'avocats, à l'information sur leurs pénibles conditions de vie par des bandes dessinées en anglais et dans les langues locales.

On voit bien, par ces quelques exemples, que de nouvelles pratiques politiques sont en train d'émerger. On voit bien qu'une nouvelle culture politique est en train de donner naissance à un autre socialisme, ou plutôt de commencer à concrétiser des socialismes qui seront différents; et ceci à partir des milieux populaires ( $y$ compris les enfants), afin qu'une démocratie véritable émerge. 
Bien sûr, nous travaillons pour le long terme. Bien sûr, "la crise, comme disait Gramsci, c'est quand le vieux n'en finit pas de mourir, alors que le neuf en est encore à naître !». Mais le neuf est en train de naître. Alors, ne dormons pas, et mettons-nous à l'ouvrage !

\section{Notes}

* Texte de la conférence prononcée par l'auteure, le 26 septembre 1985, à l'invitation de la Faculté des sciences sociales et de l'École de service social de l'Université Laval.

3 L'INODEP (Institut œecuménique pour le développement des peuples) est une organisation internationale non gouvernementale ayant statut consultatif auprès de l'UNESCO. Fondée en 1970, lors d'un colloque international de représentants d'organismes du "Tiers-Monde", elle est orientée vers la formation d'animateurs de mouvements, groupes ou organismes engagés dans des projets de développement économique, socio-politique ou culturel. Son conseil d'administration compte des représentants et représentantes d'une vingtaine de pays.

L'INODEP est une équipe pluridisciplinaire internationale, en même temps qu'un collectif autogéré qui, avec des partenaires nationaux, travaille dans des réseaux de groupes et organisations populaires. En Amérique latine, les pays actuellement touchés sont les pays du cône sud, les pays d'Amérique centrale et le Mexique. En Afrique : le Burkina Faso, le Sénégal, le Ghana, le Mali, le Zimbabwe et l'Afrique du Sud. Dans l'Océan Indien : I'île Maurice et les Seychelles. En Asie: I'Inde, le Sri Lanka, les Philippines, la Thaïlande, Singapour et la Malaisie. Dans l'Océan Pacifique: Hawai, l'Australie, la Nouvelle-Zélande, la Nouvelle-Calédonie et plusieurs petites îles. En Europe : la France, I'Angleterre, I'Italie et plusieurs autres pays en ce qui concerne le mouvement des paysans autonomes, en lien avec des mouvements paysans du Tiers-Monde. En Amérique du Nord: I'île-du-Prince-Édouard et le Québec.

Le premier président en a été Paulo Freire, philosophe et pédagogue brésilien, bien connu pour ses travaux sur l'approche de conscientisation dans l'alphabétisation et l'éducation populaire. Son président actuel est Jacques Chonchol, ancien Ministre de l'agriculture du Chili et artisan de la réforme agraire de l'Unité populaire.

L'adresse de l'INODEP est : 49, rue de la Glacière, 75013 Paris, France. 